\title{
REMARKS ON CERTAIN PATHOLOGICAL OPEN SUBSETS OF 3-SPACE AND THEIR FUNDAMENTAL GROUPS
}

\author{
W. A. BLANKINSHIP AND R. H. FOX
}

We shall consider several well known subsets of spherical 3-space $S$ : the compact zero-dimensional set $P$ described by Antoine $[1]^{1}$ and the topological 3-cell $C$ consisting of the "horned sphere" $\Sigma$ of Alexander [2] together with its interior. That the fundamental groups, $\pi_{1}(S-P)$ and $\pi_{1}(S-C)$, of their complementary domains are not finitely generated (and hence in particular not trivial) was noted by Alexander [2, pp. 9 and 11]. It is the main purpose of this note to give a

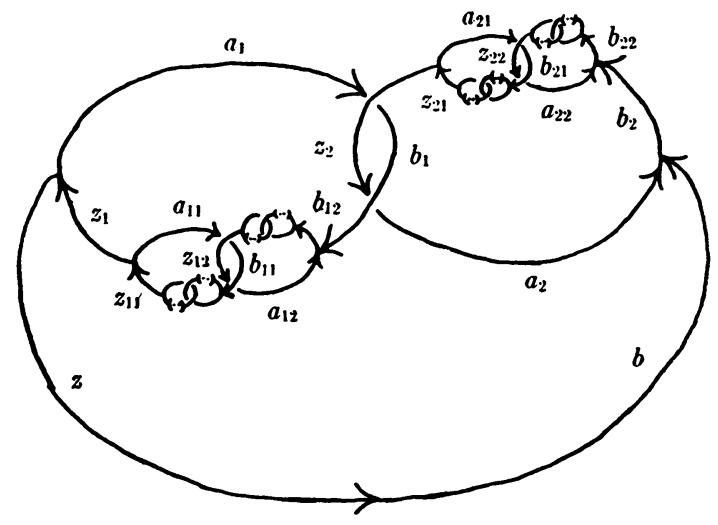

FIG. 1

complete proof of this remark, using explicit presentations of the groups. ${ }^{2}$ We feel that this is justified by the historical significance of these examples, even though on the one hand Antoine's set occurs as a special case of a recent generalization [3] to $n$-dimensional space $(n \geqq 3)$ and on the other Alexander's example has been supplanted by a much simpler one [4]. An unexpected consequence of the explicit presentation of the groups is the discovery, of which we make use, that $\pi_{1}(S-C)$ is a homomorph of $\pi_{1}(S-P)$; the geometrical meaning of this relationship between Antoine's and Alexander's examples is not evident.

Of great interest, although not as well known, is the simply con-

Received by the editors July $13,1949$.

${ }_{1}$ Numbers in brackets refer to the references cited at the end of the paper.

2 A proof that $\pi_{1}(S-P)$ is non-trivial has been given by R. P. Coelho [6]. By a presentation $\{x / r=1\}$ of a group we mean a system of generators $\left\{x_{j}\right\}$ and defining relations $\left\{r_{i}=1\right\}$. 
nected open subset $M$ of $S$, which is not a topological open 3-cell, constructed by Whitehead and Newman [5]. In \$3 we give a simplified proof that $M$ is not homeomorphic to an open 3-cell. The simplifying ideas in this proof were used [4, example 1.3] in demonstrating the properties of a certain set $Z$ which combines the essential features of the example of Alexander and that of Whitehead-Newman.

1. Alexander's horned cell. The complement, $S-C$, of Alexander's horned cell is homeomorphic to the complement of an infinite graph $\Gamma$, whose projection is shown in Fig. 1.

From this diagram a presentation of the group $\pi_{1}(S-C)=\pi_{1}(S-\Gamma)$ may be read, using a slight extension of a standard method. ${ }^{3}$ The generators are $z, z_{\alpha}, a_{\alpha}, b, b_{\alpha}$, where $\alpha$ ranges over all finite sequences of the form

$$
\alpha=\alpha_{1} \alpha_{2} \cdots \alpha_{l}, \quad \alpha_{i}=1,2, \quad l(\alpha)=l>0 .
$$

The defining relations are of three types:

(1) At the crossings (cf. Fig. 2)

$$
b_{\alpha 1}=z_{\alpha 2} a_{\alpha 1} z_{\alpha 2}^{-1}, \quad z_{\alpha 2}=b_{\alpha 1}^{-1} a_{\alpha 2} b_{\alpha 1} \text {. }
$$

FIG. 2

(2) At the points of order three (cf. Fig. 3)

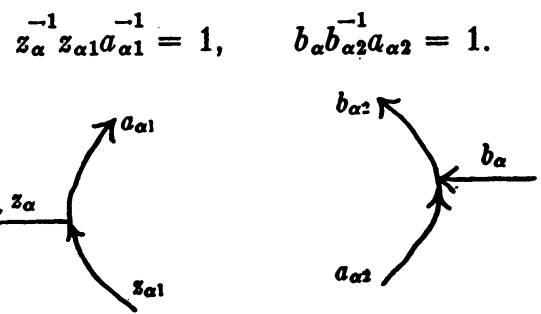

FIG. 3

(3) Around the singular points (cf. Fig. 4)

$$
b_{\alpha}=z_{\alpha},
$$

${ }^{3}$ Cf. $[4$, p. 981$]$. 
where $\alpha 1, \alpha 2$ denote the sequences of length $l+1$ formed by adjoining 1 or 2 , resp., to $\alpha$.

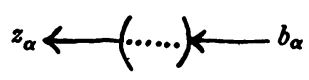

FIG. 4

Using $\left(2_{\alpha}\right)$ and $\left(3_{\alpha}\right)$ as definitions of the generators $a$ and $b$ we obtain from $\left(1_{\alpha}\right)$ the presentation: $:^{4}$

$$
G=\pi_{1}(S-C)=\left\{z_{\alpha}, l(\alpha) \geqq 0 / z_{\alpha}=\left[z_{\alpha 1}, z_{\alpha 2}^{-1}\right]\right\} .
$$

For each non-negative integer $n$ we consider the free group $G_{n}$ on generators $z_{\alpha}, l(\alpha)=n$, and define a homomorphism $\phi_{n}$ of $G_{n}$ into $G_{n+1}$ by $\phi_{n}\left(z_{\alpha}\right)=\left[z_{\alpha 1}, z_{\alpha 2}^{-1}\right]$. Clearly $G$ is the direct limit of the homomorphism sequence $G_{0} \rightarrow G_{1} \rightarrow G_{2} \rightarrow \ldots$. Moreover, for each $n, \phi_{n}$ is an isomorphism, for if $w\left(z_{\alpha}\right)$ is a reduced word in $G_{n}$, then $\phi_{n}\left(w\left(z_{\alpha}\right)\right)$ $=w\left(\left[z_{\alpha 1}, z_{\alpha 2}^{-1}\right]\right)$ is necessarily a reduced word in $G_{n+1}$. Hence the groups $G_{n}$ may be identified with certain subgroups of $G$. Thus $G=\cup_{n=0}^{\infty} G_{n}$ is the union of an increasing sequence of finitely generated free groups. Since the rank of $G_{n}$ is $2^{n}$ we obtain the result, ${ }^{5}$ $\pi_{1}(S-C)$ is a locally free group and does not have finite rank.

2. Antoine's set. Consider a solid torus $P_{0}$ in $S$ and $k$ solid tori $T_{1}, T_{2}, \cdots, T_{k}$ in $P_{0}$ arranged in cyclic order around $P_{0}$ in such a way that each torus is simply linked with each of its neighbors. Denote the union of $T_{1}, T_{2}, \cdots, T_{k}$ by $P_{1}$ and for each $i=1,2, \cdots$, $k$ select a homeomorphism $f_{i}$ of $P_{0}$ onto $T_{i}$. For any finite sequence $\alpha=\alpha_{1} \alpha_{2} \cdots \alpha_{l}, 1 \leqq \alpha_{i} \leqq k$, define $f_{\alpha}=f_{\alpha 1} f_{\alpha 2} \cdots f_{\alpha l}, T_{\alpha}=f_{\alpha}\left(P_{0}\right)$, and $P_{l}=\mathrm{U}_{l(\alpha)=l} T_{\alpha}$. Assuming $k$ sufficiently large and the tori $T_{1}, \cdots, T_{k}$ properly placed so that diam $T_{\alpha} \rightarrow 0$ as $l(\alpha) \rightarrow \infty$, the set $P=\bigcap_{l=0}^{\infty} P_{l}$ is recognizable as Antoine's set. We denote the closure of the set $P_{l}-P_{n}$ by $P_{n}^{l}$.

It is easily seen that $P_{1}^{0}$ is of the same homotopy type as the complement of the linkage whose projection is represented in Fig. 5, where we have taken $k$ even.

Labelling and orienting the edges as indicated we find by a standard procedure ${ }^{3}$ that $\pi_{1}\left(P_{1}^{0}\right)$ is generated by elements $x, u, v, w, a_{1}, b_{1}$, $\cdots, a_{k}, b_{k}$ subject to the defining relations ${ }^{4}$

4 We use the notations $[u, v]=u v u^{-1} v^{-1}, u^{v}=v u v^{-1}, u^{-v}=v u^{-1} v^{-1}$.

- To show just that $\pi_{1}(S-C)$ is non-trivial, a simpler procedure is to exhibit a representation of $G$ into the symmetric group of order 6. Cf. [3]. For definition of locally free group see [8]. 


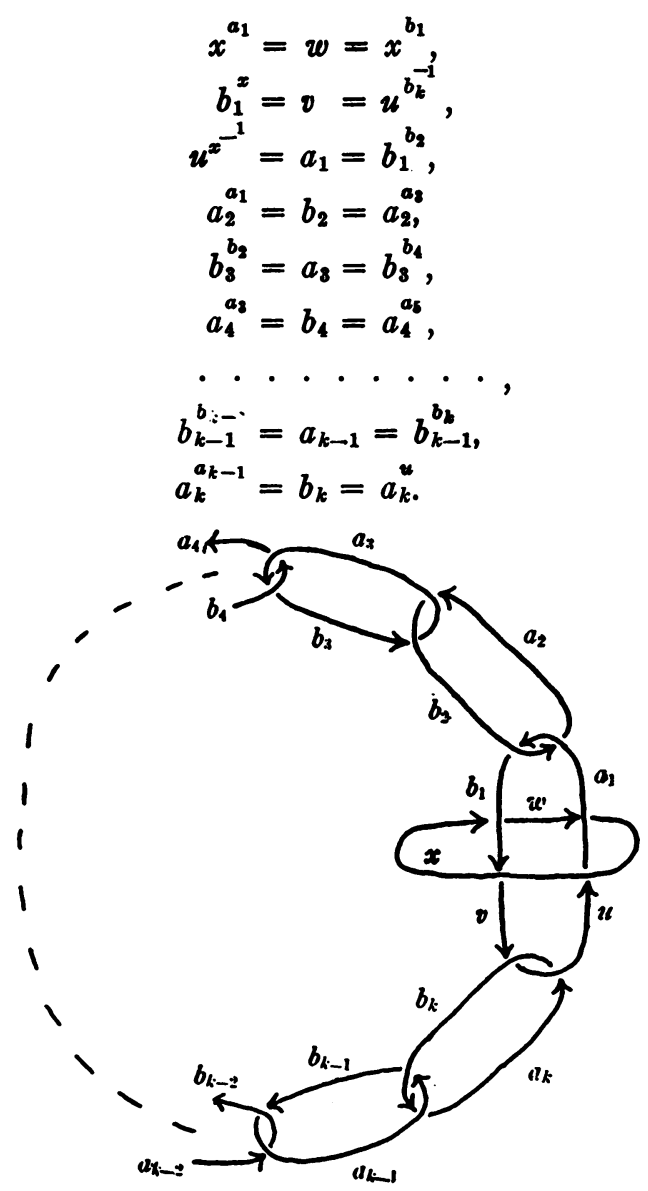

Fig. 5

From these relations we can deduce that

$$
a_{1}^{-1} b_{1}=a_{2}^{-1} b_{2}=\cdots=a_{k}^{-1} b_{k}=u^{-1} v \text {. }
$$

Denoting this element by $y$, we are now enabled to eliminate $u, v, w, b_{1}, \cdots, b_{k}$. As a result, we find that $\pi_{1}\left(P_{1}^{0}\right)$ is generated by $x, y, a_{1}, \cdots, a_{k}$, subject to the relations

$$
\begin{aligned}
{[x, y] } & =1, \\
y & =\left[\overrightarrow{a_{i}^{-1}}, a_{j-1}\right]=\left[\overrightarrow{a_{j}^{-1}}, a_{j+1}\right], \quad j=2,4,6, \cdots, k-2, \\
y & =\left[\overrightarrow{a_{k}^{-1}, a_{k-1}}\right]=\left[\overrightarrow{a_{k}^{-1}}, a_{1}^{x}\right] .
\end{aligned}
$$$$
\text { I }
$$

Now since the closure of the set $T_{i}-U_{j} T_{i j}$ is just $f_{i}\left(P_{1}^{0}\right)$, we see that its fundamental group is given by generators $x_{i}^{\prime}, y_{i}^{\prime}, a_{i 1}^{\prime}, a_{k 2}^{\prime}, \cdots, a_{i k}^{\prime}$ 
and defining relations $\mathrm{I}_{i}^{\prime}$, obtained by replacing $x, y, a_{1}, \cdots, a_{k}$ in I by $x_{i}^{\prime}, y_{i}^{\prime}, a_{i 1}^{\prime}, \cdots, a_{i k}^{\prime}$, respectively. The intersection of $P_{1}^{0}$ and $f_{i}\left(P_{1}^{0}\right)$ is the torus $\dot{T}_{i}$ which is the boundary of $T_{i}$, and whose fundamental group is free abelian on the two generators $x_{i}^{\prime}$ and $y_{i}^{\prime}$. Since $x$ and $y$ may be represented by loops bounding in the closure of $S-P_{0}$ and in $P_{0}$, respectively (see Fig. 5), it follows that $x_{i}^{\prime}=f_{i}(x)$ and $y_{i}^{\prime}=f_{i}(y)$ may be represented respectively by equatorial and meridional loops on $T_{i}$. This observation enables us to compute from Fig. 5 the relations defining the injection $\pi_{1}\left(\dot{T}_{i}\right) \rightarrow \pi_{1}\left(P_{1}^{0}\right)$. They are

$\mathrm{II}_{\boldsymbol{i}}$

$$
\begin{aligned}
y_{i} & =a_{i}, & \\
x_{i} & =a_{i+1}^{-1} a_{i-1}, & i=2,4, \cdots, k-2, \\
& =a_{i+1} a_{i-1}^{-1}, & i=3,5, \cdots, k-1, \\
& =a_{2} x^{-1} a_{k}^{-1} x, & i=1, \\
& =x a_{1}^{-1} x^{-1} a_{k-1}, & i=k,
\end{aligned}
$$

where $x_{i}, y_{i}$ (and later $a_{i 1}, \cdots, a_{i k}$ ) denote the elements represented by loops obtained by conjugating a representative of $x_{i}^{\prime}, y_{i}^{\prime}\left(a_{i 1}^{\prime}, \cdots, a_{i k}^{\prime}\right)$ by a (properly chosen) fixed path joining the base point of $\pi_{1}\left(P_{1}^{0}\right)$ to that of $\pi_{1}\left(f_{i}\left(P_{1}^{0}\right)\right)$.

Since the $k$ sets $f_{i}\left(P_{1}^{0}\right)$ are mutually disjoint, we may obtain a presentation of the group $\pi_{1}\left(P_{2}^{0}\right)$ by $k$ successive applications of the algorithm for computing the fundamental group of a union. ${ }^{6}$ We thus obtain

$$
\pi_{1}\left(P_{2}^{9}\right)=\left\{x_{\alpha}, y_{\alpha}, a_{\alpha i} / \mathrm{I}, \mathrm{I}_{i}, \mathrm{II}_{i}\right\}
$$

where $0 \leqq l(\alpha) \leqq 1, i=1,2, \cdots, k$.

Proceeding similarly with the adjunction of the sets $P_{3}^{2}, P_{4}^{3}, \cdots$, we construct inductively presentations of the groups $\pi_{1}\left(P_{n}^{0}\right)$ :

$$
\pi_{1}\left(P_{n}^{0}\right)=\left\{x_{\alpha}, y_{\alpha}, a_{\alpha i} / \mathrm{I}, \mathrm{I}_{\alpha i}, \mathrm{II}_{\alpha i}\right\}
$$

where $0 \leqq l(\alpha) \leqq n-1, i=1,2, \cdots, k$ and $\mathrm{I}_{\alpha i}, \mathrm{II}_{\alpha i}$ denote the relations obtained from $\mathrm{I}_{i}$ and $\mathrm{II}_{i}$ by replacing $i$ by $\alpha i$ (see below).

Since $\pi_{1}\left(P_{0}-P\right)$ is the direct limit of the homomorphism sequence

$$
\pi_{1}\left(P_{1}^{0}\right) \rightarrow \pi_{1}\left(P_{2}^{0}\right) \cdots,
$$

in which each homomorphism sends the generators of one group into the same-named generators of the other, we obtain $\pi_{1}\left(P_{0}-P\right)$ simply by removing the restrictions on $l(\alpha)$ in the presentations. A presentation of the group $\pi_{1}(S-P)$ is obtained by adjoining the relation $x=1$.

- Cf. $[7, \$ 52]$. 
Thus $\pi_{1}(S-P)$ is given by generators $x_{\alpha}, y_{\alpha}, a_{\alpha i}, l(\alpha) \geqq 0$, with the defining relations:

$\mathbf{I}_{\boldsymbol{\alpha}}$

$$
\begin{array}{rlr}
x & =1, \\
{\left[x_{\alpha}, y_{\alpha}\right]=1,} & \\
y_{\alpha}=\left[a_{\alpha j}^{-1}, a_{\alpha j-1}\right]=\left[a_{\alpha j}^{-1}, a_{\alpha j+1}\right], & j=2,4, \cdots, k-2, \\
y_{\alpha}=\left[a_{\alpha k}^{-1}, a_{\alpha k-1}\right]=\left[a_{\alpha k}^{-1}, a_{\alpha 1}^{z \alpha}\right], & \\
y_{\alpha i}=a_{\alpha i}, & \\
x_{\alpha i}=a_{\alpha i+1}^{-1} a_{\alpha i-1}, & \\
& =a_{\alpha i+1} a_{\alpha i-1}^{-1}, \\
& =a_{\alpha 2} x_{\alpha}^{-1} a_{\alpha k}^{-1} x_{\alpha}, \\
& =x_{\alpha} a_{\alpha 1}^{-1} x_{\alpha}^{-1} a_{\alpha k-1}, & i=2,4, \cdots, k-2, \\
\end{array}
$$

That $\pi_{1}(S-P)$ is not finitely generated (and therefore nontrivial) is demonstrated by the following homomorphism into $\pi_{1}(S-C)$ :

$$
\begin{aligned}
a_{\alpha}=y_{\alpha} & \rightarrow z_{\sigma(\alpha)}, \\
& \rightarrow z_{\sigma(\alpha)}^{-1},
\end{aligned}
$$

if $l(\alpha)$ is odd, if $l(\alpha)$ is even,

where

$$
\sigma(\alpha)=\sigma_{1} \sigma_{2} \cdots \sigma_{l}
$$$$
\sigma_{i}=1,2 \text {, }
$$

for $i=1,2, \cdots, l$ and

$$
\begin{aligned}
\sigma_{i} & \equiv \alpha_{i}(\bmod 2) \\
& \equiv 1+\alpha_{i}(\bmod 2)
\end{aligned}
$$

if $i$ is odd, if $i$ is even.

3. The Whitehead-Newman set. To obtain the Whitehead-Newman set $T_{\infty}$ one proceeds as in the construction of Antoine's set, using $k=1$ instead of $k \equiv 0(\bmod 2)$. The solid torus corresponding to $S-P_{0}$ of the preceding section is denoted in [5] by $R=S-T_{0}$. The fundamental group $G_{\infty}$ of $U_{0, \infty}=T_{0}-T_{\infty}=S-\left(R \cup T_{\infty}\right)$ is the limit of the direct homomorphism sequence

$$
G_{1} \rightarrow G_{2} \rightarrow G_{3} \rightarrow \cdots
$$

where $G_{n}$ denotes the fundamental group of $U_{0, n}=T_{0}-T_{n}=S$ $-\left(R \cup T_{n}\right)$. From the presentation (3.1) of [5] it follows that $G_{\infty}$ has the presentation

$$
\begin{array}{r}
G_{\infty}=\left\{a_{\lambda}, b_{\lambda}, 0 \leqq \lambda<\infty /\left[a_{\lambda}, b_{\lambda}\right]=1, b_{\lambda}=\left[a_{\lambda}^{-1}, b_{\lambda+1}\right]\left[a_{\lambda}^{-1}, b_{\lambda+1}^{-1}\right],\right. \\
\left.a_{\lambda+1}=\left[b_{\lambda+1}^{-1}, a_{\lambda}\right]\left[b_{\lambda+1}^{-1}, a_{\lambda}^{-1}\right]\right\} .
\end{array}
$$


The fundamental group of $S-T_{\infty}$, obtained by adjoining to $G_{\infty}$ the relation $a_{0}=1$, is obviously trivial. Thus $S-T_{\infty}$ is a simply connected open subset of $S$.

On the other hand the group $G_{\infty}$ has the representation

$$
\begin{array}{rlrl}
a_{\lambda} & \rightarrow\left(\begin{array}{lllll}
1 & 2 & 3 & 4 & 5
\end{array}\right) & & \text { (for } \lambda \text { even) } \\
& \rightarrow\left(\begin{array}{lllll}
1 & 5 & 3 & 2 & 4
\end{array}\right) & \text { (for } \lambda \text { odd) } \\
b_{\lambda} & \rightarrow\left(\begin{array}{lllll}
5 & 4 & 3 & 2 & 1
\end{array}\right) & \text { (for } \lambda \text { even) } \\
& \rightarrow\left(\begin{array}{lllll}
4 & 2 & 3 & 5 & 1
\end{array}\right) & \text { (for } \lambda \text { odd) }
\end{array}
$$

in the symmetric group of order 5 .

We assert that the complement $S-\left(T_{\infty} \cup J\right)$ of any compact subset $J$ of $S-T_{\infty}$ containing $R$ cannot be simply connected. For $S-\left(T_{\infty} \cup J\right) \subset S-\left(R \cup T_{\infty}\right) \subset U_{0, \infty}$, and we can always find $\lambda$ so large that $a_{\lambda}$ is represented by a loop in $U_{0, \infty}-J$, and this cannot be contracted to a point in $S-\left(T_{\infty} \cup J\right)$ because $a_{\lambda} \neq 1$ in $\pi_{1}\left(U_{0, \infty}\right)$. This proves immediately that $S-T_{\infty}$ is not an open 3-cell, for in an open 3-cell any compact set $R$ is contained in a closed 3-cell $J$ whose complement is simply connected.

\section{REFERENCES}

1. L. Antoine, C. R. Acad. Sci. Paris vol. 171 (1920) pp. 661-663.

1a. — J. Math. Pures Appl. (8) vol. 4 (1921) pp. 221-325.

2. J. W. Alexander, Proc. Nat. Acad. Sci. U.S.A. vol. 10 (1924) pp. 8-12.

3. W. A. Blankinship, Thesis, Princeton University, 1949.

4. R. H. Fox and Emil Artin, Ann. of Math. vol. 49 (1948) pp. 979-990.

5. M. H. A. Newman and J. H. C. Whitehead, Quart. J. Math. Oxford Ser. vol. 8 (1937) pp. 14-21.

6. R. P. Coelho, Portugaliae Mathematica vol. 6 (1947) pp. 57-65.

7. H. Seifert and W. Threlfall, Lehrbuch der Topologie.

8. A. Kurosh, C. R. (Doklady) Acad. Sci. URSS. N.S. vol. 24 (1939) pp. 99-101, or Teoriya grupp, Moscow-Leningrad, 1944, chap. 10, $\$ 48$.

Princeton University 